\title{
Fatores Associados à Autopercepção Negativa de Saúde em Idosos Assistidos Pela Atenção Básica
}

\author{
Gracielle Pampolim, ${ }^{1}$ Alessandra Miranda Ferres,${ }^{2}$ Isabelle Gadiolli Verzola, ${ }^{2}$ \\ Glenda Pereira Lima Oliveira ${ }^{2}$
}

RESUMO

O objetivo do presente estudo foi verificar a prevalência e os fatores associados à autopercepção negativa de saúde em idosos assistidos pela atenção básica em uma comunidade, em Vitória - Espírito Santo. Foi realizado um estudo observacional transversal, do tipo analítico, com 241 idosos de uma comunidade em Vitória, Espírito Santo. A autopercepção de saúde foi a variável desfecho, e as características sociodemográficas, hábitos de vida e características de saúde foram as variáveis de exposição. Os dados foram analisados a partir do teste Qui-Quadrado de Pearson e foi calculada a Razão de Prevalência para as variáveis associadas significativamente com o desfecho $(p<0,05)$. A prevalência da autopercepção negativa de saúde encontrada foi de $46,5 \%$, e os fatores associados ao desfecho foram: raça branca, polifarmácia, incapacidade para atividades cotidianas, sintomas depressivos, risco de quedas e percepção negativa da qualidade de vida. Os achados deste estudo podem auxiliar no gerenciamento do cuidado prestado aos idosos, visando à manutenção e recuperação da independência, autonomia e saúde da pessoa idosa, e assim contribuir para uma melhor qualidade de vida dessa população.

Palavras-chave: Idoso; saúde do idoso; autoavaliação; autoavaliação diagnóstica.

\section{ASSOCIATED FACTORS WITH NEGATIVE SELF-PERCEIVED HEALTH IN ELDERLY ASSISTED BY PRIMARY CARE}

\section{ABSTRACT}

The aim of the this study was to verify the prevalence and associated factors with negative self-perceived health in the elderly population assisted by primary health care in a community in Vitória - Espírito Santo. An observational, cross-sectional analytical study was carried out with 241 elderly people from a community in Vitória, Espírito Santo. Self-perceived health was the outcome variable, and sociodemographic characteristics, lifestyle and health characteristics were the exposure variables. Data were analyzed using Pearson's Chi-Square test and the Prevalence Ratio was calculated for variables significantly associated with the outcome $(p<0.05)$. The prevalence of negative self-perceived health was $46.5 \%$, and the factors associated with the outcome were: white race, polypharmacy, incapacity for daily activities, depressive symptoms, risk of falls and negative perception of quality of life. The findings of this study can assist in the management of care provided to the elderly, aiming at maintaining and recovering the independence, autonomy and health of the elderly, and thus contribute to a better quality of life for this population.

Keywords: Aged; health of the elderly; self-assessment; diagnostic self evaluation.

RECEBIDO EM: 1\%/6/2020

MODIFICAÇÕES SOLICITADAS EM: 6/7/2020

ACEITO EM: 13/8/2021

\footnotetext{
${ }^{1}$ Autora correspondente. Escola Superior de Ciências da Santa Casa de Misericórdia de Vitória. Av. Nossa Senhora da Penha, 2190 - Santa Luíza. Vitória/ES, Brasil. CEP 29045-402. http://lattes.cnpq.br/1398939161667908. https://orcid.org/00000002-4157-3521. graciellepampolim@hotmail.com

2 Escola Superior de Ciências da Santa Casa de Misericórdia de Vitória. Vitória/ES, Brasil.
} 


\section{INTRODUÇÃO}

Nos últimos 20 anos a expectativa de vida da população aumentou consideravelmente, fato que pode ser atribuído ao avanço tecnológico significativo na área da saúde em todo o mundo. ${ }^{1}$ No Brasil, segundo dados do Instituto Brasileiro de Geografia e Estatística - IBGE - em termos quantitativos, os idosos estão crescendo mais do que os outros segmentos etários, e atualmente, representam $13 \%$ da população brasileira total. ${ }^{2}$

Atrelado a isso percebe-se também um aumento crescente de doenças crônicas que se concentram majoritariamente nessa população. Os principais agravos crônicos comuns à população idosa são a hipertensão arterial sistêmica e o diabetes mellitus que, juntos, contribuem para o surgimento de importantes complicações à saúde do idoso, tais como: complicações renais, cardiovasculares e cerebrovasculares, podendo interferir na autonomia e independência do idoso e consequentemente influenciar na forma como esse indivíduo percebe sua saúde e qualidade de vida.,

Neste contexto, a utilização de ferramentas que permitam estimar e acompanhar as condições de saúde destes indivíduos é de grande importância. Uma dessas ferramentas é a autopercepção de saúde, uma medida subjetiva, que outrora era estudada como uma variável de exposição, mas atualmente é vista como provável preditora de desfechos funcionais, psicológicos e até de mortalidade, e, por isso, tem sido considerada um valioso indicador de saúde para a pessoa idosa. ${ }^{5}$

Estudos publicados recentemente apontam para prevalências entre 41,1\% e 53,7\% de autopercepção positiva de saúde entre os idosos estudados., ${ }^{6,7}$ E destacam fatores como a boa interação com familiares e amigos, além da prática de atividades para lazer como importantes fatores associados à manutenção da qualidade de vida e da percepção do estado geral de saúde do idoso. ${ }^{8}$

Enquanto que a autopercepção negativa de saúde parece estar fortemente associada com fatores como gênero feminino, mobilidade reduzida, multimorbidades, polifarmácia, histórico de internações, ausência de vínculo trabalhista, sintomas depressivos e dependência funcional para as atividades básicas de vida diária. 5,9,10

Diante do exposto, percebe-se que além de contribuir para o levantamento de inúmeras situações adversas da saúde dos idosos, a autopercepção pode ainda auxiliar na fundamentação de ações e programas voltados para este estrato populacional. Desse modo, este estudo tem por objetivo verificar a prevalência e os fatores associados à autopercepção negativa de saúde em idosos assistidos pela atenção básica em uma comunidade em Vitória - Espírito Santo.

\section{MÉTODO}

Trata-se de um estudo observacional transversal, de abordagem quantitativa, realizado com idosos assistidos por uma Unidade de Saúde da Família (USF) da cidade de Vitória, capital do Espírito Santo. A amostra foi composta por indivíduos com idade igual ou superior a 60 anos, adscritos ao território da referida

Editora Unijuí - Revista Contexto \& Saúde - ISSN 2176-7114 - v. 21, n. 44, out./dez. 2021 
USF, que aceitaram participar da pesquisa mediante assinatura do Termo de Consentimento Livre e Esclarecido.

A amostra foi composta por 241 idosos, cujo cálculo amostral deu-se a partir do número total de idosos cadastrados no território da USF estudada em 2018, com margem de erro de 0,05, estimativa de proporção de 0,5 e acréscimo de $30 \%$ para possíveis perdas amostrais. A seleção foi realizada de forma aleatória simples, na qual os idosos foram ordenados alfabeticamente e de acordo com a microárea em que residiam; e, então, foram sorteados aleatoriamente dois em cada três destes indivíduos. As entrevistas e avaliações tiveram lugar na residência destes idosos, em horário predeterminado e por avaliadores treinados para tal, entre abril e junho de 2018. Foram excluídos os indivíduos que não possuíam condições de responder ao questionário ou não dispunham de cuidador apto para tal, quando houve impossibilidade de acesso por recusa ou restrição da família, em casos de migração para outra região ou óbito, prévios à realização das entrevistas.

As análises foram realizadas segundo a variável dependente autopercepção de saúde, avaliada por meio do questionamento "Como o(a) Sr(a) classificaria seu estado de saúde?", tendo como opções de resposta: Muito bom, Bom, Regular, Ruim e Muito ruim. Para as análises, as opções foram dicotomizadas em autopercepção positiva (Muito bom/Bom), e negativa (Regular/Ruim/Muito ruim), seguindo o adotado na literatura. ${ }^{11}$ As variáveis independentes foram: características sociodemográficas e hábitos de vida (faixa etária, sexo, etnia, escolaridade, restrição ao lar, residência multigeracional, hábitos alcoólicos, tabagismo, prática de atividade física e de lazer), e características de saúde (presença de multimorbidade, polifarmácia, incapacidade para realização de atividades básicas e instrumentais de vida diária, sintomas depressivos, risco de quedas e qualidade de vida).

A polifarmácia é uma variável que pode ser definida como o uso de cinco ou mais medicamentos prescritos por um profissional de saúde habilitado, considerando os riscos e benefícios individualmente e a real necessidade clínica para cada paciente. ${ }^{12,13}$ Os sintomas depressivos estabeleceram-se por meio da Escala Geriátrica de Depressão (GDS), que é composta por 15 perguntas afirmativas/ negativas. O ponto de corte adotado nesta pesquisa foi $5 / 6$, na qual o resultado igual a 6 ou mais pontos determinam uma sintomatologia depressiva. ${ }^{14}$

O Índice de Katz, instrumento que apresenta boa consistência interna e foi traduzido e validado para o idioma brasileiro, ${ }^{15}$ é utilizado para avaliar seis atividades básicas de vida diária: vestir-se, tomar banho, utilizar o banheiro, movimentar-se, continência dos esfíncteres e alimentar-se. Já o Índice de Lawton e Brody, traduzido e validado para o contexto brasileiro, ${ }^{16}$ mostra-se confiável para avaliar a realização de atividades instrumentais de vida diária, tais como utilizar o telefone, fazer compras, preparar o próprio alimento, realizar tarefas domésticas, usar os meios de transporte, gerenciar o uso de medicamentos e administrar o próprio dinheiro. ${ }^{17}$ Nessa análise são considerados idosos dependentes aqueles que relataram incapacidade para realização de uma ou mais atividades descritas, para ambas as escalas.

Editora Unijuí - Revista Contexto \& Saúde - ISSN 2176-7114 - v. 21, n. 44, out./dez. 2021 
Para estimar o risco de quedas foi utilizada a Escala de Tinetti, validada e traduzida para o Português, ${ }^{18}$ que avalia a marcha e equilíbrio de idosos e cuja pontuação varia de 0 a 28 pontos, sendo adotado como ponto de corte valor igual ou menor que 24 pontos que indicam de moderado a alto risco de quedas. ${ }^{19}$ Para analisar a qualidade de vida foi usado o Questionário de Avaliação de Qualidade de Vida (Brasil SF-36), um instrumento validado e traduzido para a população brasileira, ${ }^{20}$ composto por 36 itens referentes à saúde que podem resultar entre 0 e 100 pontos $^{21}$ que foram divididos em quartis, em que o 3ㅇ quartil, equivalente à pontuação 75 ou mais, foi adotado como indicativo de percepção positiva de qualidade de vida.

A análise de dados foi realizada de forma descritiva e inferencial, para a qual foi adotado o teste Qui-Quadrado de Pearson, considerando nível de significância de 0,05 e Intervalo de Confiança de 95\%, e o cálculo da Razão de Prevalência foi adotado para as variáveis com significância estatística de $p<0,05$.

Este estudo foi apresentado e autorizado pelo Comitê de Ética em Pesquisa da Escola Superior de Ciências da Santa Casa de Misericórdia de Vitória, sob no 2.142.377. Todos os participantes assinaram o Termo de Consentimento Livre e Esclarecido e em todas as fases da pesquisa foram aplicados e reconhecidas os preceitos estipulados nas Diretrizes e Normas Regulamentadoras de Pesquisa Envolvendo Seres Humanos, da Resolução 466/12.

\section{RESULTADOS}

A prevalência de autopercepção de saúde Muito boa/Boa foi de 53,5\% (IC 95\%: 46,9-60,2); 46,5\% autoperceberam sua saúde como Regular/Ruim ou Muito Ruim (IC 95\%: 39,8 - 53,1) (dados não apresentados em tabela).

O perfil dos idosos estudados consistiu majoritariamente em idosos jovens, do sexo feminino, negros ou pardos, de baixa escolaridade, sem restrição ao convívio social, coabitando em residência multigeracional, sem hábitos alcoólicos ou tabagismo, que não praticam atividade física, mas realizam alguma atividade de lazer. Com relação às condições de saúde, em sua maioria os idosos apresentavam multimorbidade e percepção negativa da qualidade de vida e não apresentavam polifarmácia, sintomas depressivos, risco de quedas ou incapacidade para as atividades de vida diária (Tabela 1 ).

Tabela 1 - Caracterização de idosos de acordo com variáveis sociodemográficas, hábitos de vida e condições de saúde. Unidade de Saúde de Jesus de Nazareth, Vitória-ES

\begin{tabular}{lcccc}
\hline \multirow{2}{*}{ Variáveis } & \multicolumn{3}{c}{$\begin{array}{c}\text { Amostra total } \\
\mathbf{n = 2 4 1}\end{array}$} & \multirow{2}{*}{ IC 95\% } \\
\cline { 2 - 3 } & $\boldsymbol{n}$ & $\mathbf{( \% )}$ & \\
\cline { 1 - 3 } Faixa Etária & 174 & $(72,2)$ & $66,4-78,0$ \\
60 a 74 anos & 67 & $(27,8)$ & $22,0-33,6$ \\
75 anos ou mais & & & \\
Sexo & 93 & $(38,6)$ & $32,4-44,4$ \\
Masculino & 148 & $(61,4)$ & $55,6-67,6$ \\
Feminino & & & \\
& & &
\end{tabular}

Editora Unijuí - Revista Contexto \& Saúde - ISSN 2176-7114 - v. 21, n. 44, out./dez. 2021 


\section{Etnia}

Branco(a)

Negro(a)/Pardo(a)

Escolaridade

0 a 4 anos de estudo

5 anos ou mais de estudo

\section{Restrito ao lar}

Sim

Não

Residência multigeracional

Sim

Não

Hábitos alcoólicos

Sim

Não

Hábitos tabágicos

Sim

Não

\section{Atividade física}

Sim

Não

Atividade de lazer

Sim

Não

Multimorbidade

Sim

Não

Polifarmácia

Sim

Não

Incapacidade para ABVD

Sim

Não

Incapacidade para AIVD

Sim

Não

Sintomas depressivos

Sim

Não

Risco de Quedas

Sim

Não

Qualidade de vida

Positiva

Negativa

USF - Unidade de Saúde da Família; ABVD - Atividades Básicas de Vida Diária; AIVD - Atividades Instrumentais de Vida Diária; Os totais de determinadas variáveis não somam 241 idosos em razão da incompletude dos dados.

Fonte: Elaborada pelos autores

\begin{tabular}{|c|c|c|}
\hline 62 & $(25,7)$ & $20,3-30,7$ \\
\hline 179 & $(74,3)$ & $69,3-79,7$ \\
\hline 140 & $(58,1)$ & $51,9-63,9$ \\
\hline 101 & $(41,9)$ & $36,1-48,1$ \\
\hline 48 & $(20,0)$ & $15,0-25,4$ \\
\hline 192 & $(80,0)$ & $74,6-85,0$ \\
\hline 129 & $(54,4)$ & $48,1-60,8$ \\
\hline 108 & $(45,6)$ & $39,2-51,9$ \\
\hline 53 & $(22,1)$ & $17,1-27,5$ \\
\hline 187 & $(77,9)$ & $72,5-82,9$ \\
\hline 31 & $(13,0)$ & $8,8-17,2$ \\
\hline 207 & $(87,0)$ & $82,8-91,2$ \\
\hline 78 & $(32,5)$ & $26,7-38,7$ \\
\hline 162 & $(67,5)$ & $61,3-73,3$ \\
\hline 150 & $(62,2)$ & $55,6-68,0$ \\
\hline 91 & $(37,8)$ & $32,0-44,4$ \\
\hline 156 & $(64,7)$ & $58,1-71,0$ \\
\hline 85 & $(35,3)$ & $29,0-41,9$ \\
\hline 86 & $(35,7)$ & $30,3-41,5$ \\
\hline 155 & $(64,3)$ & $58,5-69,7$ \\
\hline 83 & $(34,4)$ & $28,2-40,2$ \\
\hline 158 & $(65,6)$ & $59,8-71,8$ \\
\hline 82 & $(34,0)$ & $27,0-40,2$ \\
\hline 159 & $(66,0)$ & $59,8-73,0$ \\
\hline 59 & $(24,5)$ & $18,7-29,9$ \\
\hline 182 & $(75,5)$ & $70,1-81,3$ \\
\hline 119 & $(49,4)$ & $43,2-56,0$ \\
\hline 122 & $(50,6)$ & $44,0-56,8$ \\
\hline 73 & $(42,7)$ & $34,5-50,3$ \\
\hline 98 & $(57,3)$ & $49,7-65,5$ \\
\hline
\end{tabular}


Nas análises bivariadas entre a autopercepção de saúde e as características sociodemográficas e hábitos de vida foi encontrado associação estatisticamente significante para a variável etnia (Tabela 2).

Tabela 2 - Distribuição das prevalências de características sociodemográficas e hábitos de vida de idosos, segundo autopercepção de saúde. Unidade de Saúde de Jesus de Nazareth, Vitória-ES

\begin{tabular}{|c|c|c|c|c|c|}
\hline \multirow[t]{2}{*}{ Variáveis } & \multicolumn{2}{|c|}{$\begin{array}{c}\text { Autopercepção } \\
\text { Negativa } \\
\mathrm{n}=112 \\
\end{array}$} & \multicolumn{2}{|c|}{$\begin{array}{c}\text { Autopercepção } \\
\text { Positiva } \\
\text { n=129 }\end{array}$} & \multirow[t]{2}{*}{$p$-valor } \\
\hline & $n$ & (\%) & $n$ & (\%) & \\
\hline \multicolumn{6}{|l|}{ Faixa Etária } \\
\hline 60 a 74 anos & 80 & $(46,0)$ & 94 & $(54,0)$ & \multirow{2}{*}{0,804} \\
\hline 75 anos ou mais & 32 & $(47,8)$ & 35 & $(52,2)$ & \\
\hline \multicolumn{6}{|l|}{ Sexo } \\
\hline Masculino & 40 & $(43,0)$ & 53 & $(57,0)$ & \multirow{2}{*}{0,393} \\
\hline Feminino & 72 & $(48,6)$ & 76 & $(51,4)$ & \\
\hline \multicolumn{6}{|l|}{ Etnia } \\
\hline Branco(a) & 38 & $(61,3)$ & 24 & $(38,7)$ & \multirow{2}{*}{0,007} \\
\hline Negro(a)/Pardo(a) & 74 & $(41,3)$ & 105 & $(58,7)$ & \\
\hline \multicolumn{6}{|l|}{ Escolaridade } \\
\hline 0 a 4 anos de estudo & 58 & $(41,4)$ & 82 & $(58,6)$ & \multirow{2}{*}{0,065} \\
\hline 5 anos ou mais de estudo & 54 & $(53,5)$ & 47 & $(46,5)$ & \\
\hline \multicolumn{6}{|l|}{ Restrito ao lar } \\
\hline Sim & 28 & $(58,3)$ & 20 & $(41,7)$ & \multirow{2}{*}{0,070} \\
\hline Não & 84 & $(43,8)$ & 108 & $(56,2)$ & \\
\hline \multicolumn{6}{|l|}{ Residência multigeracional } \\
\hline Sim & 62 & $(48,1)$ & 67 & $(51,9)$ & \multirow{2}{*}{0,788} \\
\hline Não & 50 & $(46,3)$ & 58 & $(53,7)$ & \\
\hline \multicolumn{6}{|l|}{ Hábitos alcoólicos } \\
\hline Sim & 24 & $(45,3)$ & 29 & $(54,7)$ & \multirow{2}{*}{0,819} \\
\hline Não & 88 & $(47,1)$ & 99 & $(52,9)$ & \\
\hline \multicolumn{6}{|l|}{ Hábitos tabágicos } \\
\hline Sim & 16 & $(51,6)$ & 15 & $(48,4)$ & \multirow{2}{*}{0,518} \\
\hline Não & 94 & $(45,4)$ & 113 & $(54,6)$ & \\
\hline \multicolumn{6}{|l|}{ Atividade física } \\
\hline Sim & 31 & $(39,7)$ & 47 & $(60,3)$ & \multirow{2}{*}{0,136} \\
\hline Não & 81 & $(50,0)$ & 81 & $(50,0)$ & \\
\hline \multicolumn{6}{|l|}{ Atividade de lazer } \\
\hline $\operatorname{Sim}$ & 68 & $(45,3)$ & 82 & $(54,7)$ & \multirow{2}{*}{0,649} \\
\hline Não & 44 & $(48,4)$ & 47 & $(51,6)$ & \\
\hline
\end{tabular}

Com relação às condições de saúde, a autopercepção de saúde esteve associada com polifarmácia, incapacidade para atividades básicas e instrumentais de vida diária, sintomas depressivos, risco de quedas e qualidade de vida (Tabela 3). 
Tabela 3 - Distribuição das prevalências das condições de saúde de idosos, segundo autopercepção de saúde. Unidade de Saúde de Jesus de Nazareth, Vitória-ES

\begin{tabular}{|c|c|c|c|c|c|}
\hline \multirow[t]{2}{*}{ Variáveis } & \multicolumn{2}{|c|}{$\begin{array}{c}\text { Autopercepção } \\
\text { Negativa } \\
\mathrm{n}=\mathbf{1 1 2}\end{array}$} & \multicolumn{2}{|c|}{$\begin{array}{c}\text { Autopercepção } \\
\text { Positiva } \\
\text { n=129 }\end{array}$} & \multirow[t]{2}{*}{$p$-valor } \\
\hline & $n$ & $(\%)$ & $n$ & $(\%)$ & \\
\hline \multicolumn{6}{|c|}{ Multimorbidade } \\
\hline $\operatorname{sim}$ & 74 & $(47,4)$ & 82 & $(52,6)$ & \multirow{2}{*}{0,685} \\
\hline Não & 38 & $(44,7)$ & 47 & $(55,3)$ & \\
\hline \multicolumn{6}{|c|}{ Polifarmácia } \\
\hline $\operatorname{sim}$ & 51 & $(59,3)$ & 35 & $(40,7)$ & \multirow{2}{*}{0,003} \\
\hline Não & 61 & $(39,4)$ & 94 & $(60,6)$ & \\
\hline \multicolumn{6}{|c|}{ Incapacidade para ABVD } \\
\hline Sim & 49 & $(59,0)$ & 34 & $(41,0)$ & \multirow{2}{*}{0,005} \\
\hline Não & 63 & $(39,9)$ & 95 & $(60,1)$ & \\
\hline \multicolumn{6}{|c|}{ Incapacidade para AIVD } \\
\hline Sim & 49 & $(59,8)$ & 33 & $(40,2)$ & \multirow{2}{*}{0,003} \\
\hline Não & 63 & $(39,6)$ & 96 & $(60,4)$ & \\
\hline \multicolumn{6}{|c|}{ Sintomas depressivos } \\
\hline Sim & 47 & $(79,7)$ & 12 & $(20,3)$ & \multirow{2}{*}{$<0,001$} \\
\hline Não & 65 & $(35,7)$ & 117 & $(64,3)$ & \\
\hline \multicolumn{6}{|c|}{ Risco de Quedas } \\
\hline Sim & 65 & $(54,6)$ & 54 & $(45,4)$ & \multirow{2}{*}{0,012} \\
\hline Não & 47 & $(38,5)$ & 75 & $(61,5)$ & \\
\hline \multicolumn{6}{|c|}{ Qualidade de vida } \\
\hline Positiva & 17 & $(23,3)$ & 56 & $(76,7)$ & \multirow{2}{*}{$<0,001$} \\
\hline Negativa & 64 & $(65,3)$ & 34 & $(34,7)$ & \\
\hline
\end{tabular}

ABVD - Atividades Básicas de Vida Diária; AIVD - Atividades Instrumentais de Vida Diária.

Os totais de determinadas variáveis não somam 241 idosos em razão da incompletude dos dados.

Fonte: Elaborada pelos autores.

Na Tabela 4 estão descritos os valores de Razão de Prevalência das variáveis que se mostraram associadas à autopercepção negativa de saúde nos idosos estudados.

Tabela 4 - Razão de prevalência de autopercepção negativa de saúde em idosos, segundo características sociodemográficas, hábitos de vida e condições de saúde. Unidade de Saúde de Jesus de Nazareth, Vitória-ES

\begin{tabular}{lccc}
\hline \multicolumn{1}{c}{ Variáveis Independentes } & RP & IC 95\% & p-valor \\
\hline Raça branca & 2,2 & $1,24-4,06$ & 0,007 \\
Apresentar polifarmácia & 1,5 & $1,12-1,98$ & 0,003 \\
Presença de incapacidade para ABVD & 1,5 & $1,20-1,96$ & 0,005 \\
Presença de incapacidade para AIVD & 1,5 & $1,12-2,00$ & 0,003 \\
Presença de sintomas depressivos & 3,2 & $1,89-5,30$ & $<0,001$ \\
Presença de risco de quedas & 1,4 & $1,07-1,72$ & 0,012 \\
Percepção negativa da qualidade de vida & 6,2 & $3,13-11,28$ & $<0,001$ \\
\hline
\end{tabular}

ABVD - Atividades Básicas de Vida Diária; AIVD - Atividades Instrumentais de Vida Diária; RP - Razão de Prevalência; IC - Intervalo de Confiança.

Fonte: Elaborada pelos autores. 


\section{DISCUSSÃO}

A prevalência de autopercepção regular/negativa de saúde identificada neste trabalho foi de $46,5 \%$ (IC 95\%: 39,8 - 53,1), similar à prevalência de $46,3 \%$ encontrada por um estudo ${ }^{7}$ ao estudarem idosos também assistidos pela atenção básica, no entanto menor do que os 57,5\% de autopercepção negativa de saúde encontrada por outra pesquisa ${ }^{11}$. Importante destacar que outra análise chama a atenção ainda para uma importante variação nas prevalências encontradas na literatura, podendo oscilar entre $12,6 \%$ e 51,9\% ${ }^{5}$. Essa oscilação pode ser explicada devido à categorização entre positiva, regular e negativa ser distinta entre os estudos, além da discrepância entre as regiões consideradas para as pesquisas.

Com relação às características associadas a este desfecho, verificou-se que há uma forte relação entre a autopercepção de saúde e a raça: os idosos autodeclarados brancos foram 2,2 vezes mais prevalentes (IC 95\%: 1,24-4,06; $p$ $=0,007)$ no grupo com autopercepção negativa de saúde, o que se contrapõe aos dados encontrados no estudo que analisou os idosos incluídos na base de dados da Pesquisa Nacional de Amostra por Domicílios, em que os idosos autodeclarados pardos apresentaram probabilidade $11 \%$ maior de autorreferirem sua saúde de forma negativa (OR = 1,11; IC95\%: 1,03-1,18) do que os da raça branca. ${ }^{22}$ Cabe ressaltar, porém, que os idosos incluídos no presente estudo são majoritariamente idosos de classe social inferior, por isso entende-se que a população branca no território estudado poderia apresentar fatores preditores não condizentes com o padrão da sociedade atual, culminando, assim, em uma autopercepção negativa do estado de saúde geral.

No presente estudo verificou-se que os idosos que autopercebem sua saúde de forma negativa eram 50\% mais prevalentes (RP: 1,5; IC 95\%: 1,12 1,$98 ; p=0,003$ ) entre aqueles que estão em uso de polifarmácia, corroborando achados que mostraram em suas análises importante associação entre polifarmácia e autopercepção de saúde e que mais de $50 \%$ dos idosos estudados, com polifarmácia, autoperceberam sua saúde de forma negativa. ${ }^{12}$ Uma justificativa para essa associação reside no fato de que ao se verem diante da necessidade de tomar tantos medicamentos diariamente, e ainda precisarem lidar com os processos patológicos das doenças para as quais os medicamentos são receitados, pode fazer com que o idoso adote a percepção de que está muito doente, o que consequentemente vai afetar sua percepção de saúde e qualidade de vida.

Outro achado deste estudo diz respeito às atividades de vida diária, em que os idosos com autopercepção negativa de saúde apresentaram 1,5 vezes mais prevalência de incapacidade para a realização das atividades básicas e instrumentais de vida diária (IC 95\%: 1,20 - 1,96; p = 0,005; IC 95\%: 1,12 - 2,00; $p$ $=0,003$, respectivamente). De acordo com a literatura, o processo de redução das capacidades funcionais do idoso geralmente está associado também ao aumento das comorbidades e gradativa diminuição de sua autonomia quanto às tarefas cotidianas, repercutindo negativamente na sua percepção de saúde. ${ }^{23,24}$ Além disso, de acordo com a literatura, as atividades com maiores índices de dependências são aquelas que precisam de equilíbrio, destreza e força muscular,

Editora Unijuí - Revista Contexto \& Saúde - ISSN 2176-7114 - v. 21, n. 44, out./dez. 2021 


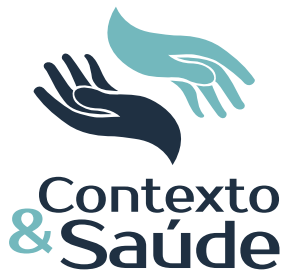

como vestir-se e banhar-se sozinhos, e continência de esfíncteres, explicada pela perda do tônus muscular no processo de envelhecimento natural. ${ }^{25,26}$

Todos estes aspectos, em especial a dificuldade de controle de esfíncteres pode fazer com que o idoso venha cada vez mais se abster do convívio social e familiar, por vergonha ou desconforto, e por isso pode não procurar ajuda, e acaba por entrar em um ciclo vicioso, tornando-se progressivamente dependente, como mostra outro estudo, ${ }^{27}$ que aponta altas prevalências de dependência funcional entre os idosos restritos ao lar avaliados.

Outro agravante da saúde dos idosos é a presença de sintomas depressivos, que por vezes pode fazer com que o indivíduo perceba sua saúde de forma mais negativa do que ela realmente é. No presente estudo os idosos com sintomas depressivos foram 3,2 vezes mais prevalentes entre aqueles com autopercepção negativa de saúde (IC 95\%: 1,89-5,30; p < 0,001). Semelhante a este dado, um estudo realizado com 360 idosos assistidos em um centro de referência, ${ }^{28}$ também encontrou uma importante associação entre as prevalências de autopercepção de saúde e sintomas depressivos. É notório que os sintomas depressivos geram sentimento de insegurança e negatividade, o que colabora para uma percepção negativa do indivíduo sobre sua saúde, que pode, desta forma, afetar fortemente o convívio social desses sujeitos, bem como o modo como lidam com sua própria saúde.

Com relação ao risco de quedas, averiguou-se que há uma importante relação entre risco de quedas e a autopercepção negativa de saúde, em que aqueles idosos com risco de queda foi $40 \%$ mais prevalente (RP: 1,4; IC 95\%: 1,07 $-1,72 ; p=0,012$ ) entre aqueles que autoavaliaram sua saúde de forma negativa. Tal fato condiz com os dados encontrados por uma pesquisa que analisou os fatores associados à recorrência de quedas de idosos em uma comunidade e obteve como resultado uma associação entre a baixa autopercepção de saúde e a recorrência de quedas ${ }^{29}$. Nesse sentido, entende-se que com a presença do risco de quedas, e um histórico por vezes recorrente, podem culminar em sentimentos de medo e insegurança, e também na perda de independência nas atividades de vida diária (AVD), o que contribui para que estes idosos tornem-se cada vez mais estagnados, podendo afetar assim tanto a saúde física quanto mental e social desses indivíduos.

Por fim, outra importante característica analisada neste estudo foi a percepção da qualidade de vida dos idosos, que se mostrou fortemente associada com a autopercepção de saúde. Os idosos que apresentavam percepção negativa da qualidade de vida foram 6,2 vezes mais prevalentes (IC 95\%: 3,13-11,28; $p$ $<0,001)$ no grupo de idosos que percebiam sua saúde de forma negativa. SemeIhante a este achado, um estudo ${ }^{30}$ realizado em Curitiba constatou uma chance três vezes maior da presença de uma percepção de saúde mais negativa naquelas idosas que obtiveram uma pontuação negativa no domínio físico do questionário de qualidade de vida (OR = 3,86, IC95\%: 2,23-6,68; $p<0,001$ ), bem como aquelas que possuíram uma percepção negativa do domínio do meio ambiente (OR = 3,50, IC95\%: 2,07-5,90; $p<0,001$ ), relações sociais (OR = 2,80, IC95\%: 1,69-4,68; $p<0,001$ ) e psicológico ( $O R=3,58, I C 95 \%: 2,17-5,92 ; p<0,001)$. Em relação ao escore médio dos quatro domínios, é possível observar uma chance de 3,69\% 
(OR $=3,69$, IC95\%: 2,13-6,39; $p<0,001)$ de as idosas que obtiveram um somatório de pontos negativo apresentarem uma autopercepção menos positiva de saúde. O que não se configura como surpresa, uma vez que, ao perceber sua saúde de forma negativa, o idoso tende a fazer o mesmo com outros aspectos de sua vida, refletindo assim na sua qualidade de vida em geral.

Os achados do presente estudo são importantes para auxiliar no melhor entendimento desse complexo e multifatorial universo que é a saúde da pessoa idosa. Acredita-se que tais achados podem contribuir para o gerenciamento do cuidado dessa população, visando sempre à prevenção de agravos que podem resultar em uma percepção negativa de saúde. Para tal, indica-se que as Unidades de Saúde que assistem essa população disponibilizem atividades com o intuito de manter e recuperar a independência e autonomia dos idosos, bem como se apropriar do estímulo à prática de atividades físicas diversas que podem auxiliar no controle de doenças crônicas e até mesmo reduzir a necessidade da utilização sistemática de medicação.

Importante salientar que limitações devem ser consideradas ao se analisar os resultados. A principal delas é a limitação inerente aos estudos transversais que resultam na impossibilidade de estabelecimento de relação causal entre desfecho e exposição. E, por fim, vale considerar também como possível limitação a dificuldade de se obter resultados totalmente precisos em razão das possíveis omissões nos relatos dos idosos, porém, como forma de evitar tal limitação, é importante esclarecer que as entrevistas foram realizadas de forma individual, em ambiente reservado, visando ao maior conforto dos idosos.

\section{CONCLUSÃO}

De acordo com os resultados obtidos, a autopercepção negativa mostrou-se prevalente na população, além de estar associada a vários fatores, sendo eles a raça branca, polifarmácia, incapacidade na realização das atividades de vida diária, sintomas depressivos, risco de quedas e percepção negativa da qualidade de vida.

Com isso, entende-se que as condições socioeconômicas, relações interpessoais e comportamentais, além de aspectos psicológicos e ambientais, podem estar interligadas e serem fatores preditores desta autoavaliação de saúde. Desse modo, o vínculo pessoal, social, trabalhista e ambiental mostra-se importante para uma concepção de saúde consideravelmente boa ou ótima.

\section{REFERÊNCIAS}

1 Matheres CD, Stevens GA, Boerma T, White RA, Tobias MI. Causes of international increases in older age life expectancy. Lancet. [internet]. 2015 [citado 10 ago. 2021];385(9967):540-548. doi: https://doi.org/10.1016/S0140-6736(14)60569-9. [Acesso em: 3 nov. 2021].

2 Instituto Brasileiro de Geografia e Estatística. Longevidade, viver bem e cada vez mais. Rev. Retratos. [internet]. 2019 [citado 10 ago. 2021];(16):20-28. Disponível em: https://agenciadenoticias.ibge.gov.br/media/com_mediaibge/arquivos/d4581e6bc87ad8768073f974c0a1102b.pdf. [Acesso em: 3 nov. 2021]. 
${ }^{3}$ Ansah JP, Inn RLH, Ahmad S. An evaluation of the impact of aggressive hypertension, diabetes and smoking cessation management on CVD outcomes at the population level: a dynamic simulation analysis. BMC Public Health. [internet]. 2019 [citado 10 ago. 2021]; 19(1105). doi: https://doi.org/10.1186/s12889-019-7429-2. Acesso em: 3 nov. 2021.

4. Dantas IC, Pinto Junior EP, Medeiros KKA, Souza EA. Perfil de morbimortalidade e os desafios para a atenção domiciliar do idoso brasileiro. Rev. Kairós Gerontol. [Internet]. 2017 [citado 10 ago. 2021];20(1):93-108. doi: https://doi.org/10.23925/2176-901X. 2017v20i1p93-108. Acesso em: 3 nov. 2021.

${ }^{5}$ Pagotto V, Bachion MM, Silveira EA. Autoavaliação da saúde por idosos brasileiros: revisão sistemática da literatura. Rev. Panam. Salud Publica. [Internet]. 2013 [citado ago. 2021;33(4):302-310. Disponível em: https://www.scielosp.org/article/rpsp/2013. v33n4/302-310/

${ }^{6}$ Souza MS, Coqueiro RS, Fernandes MH. Estudo populacional sobre os determinantes da autopercepção de saúde de idosos residentes em comunidade. Cienc. Enferm. [Internet]. 2016 [citado 10 ago. 2021];22(2):13-26. Doi: http://dx.doi.org/10.4067/S071795532016000200002. Acesso em: 3 nov. 2021.

7 Poubel PB, Lemos ELC, Araújo FC, Leite GC, Freitas IS, Silva RMA et al. Autopercepção de saúde e aspectos clínico-funcionais dos idosos atendidos em uma unidade básica de saúde no norte do Brasil. J. Health Biol. Sci. [internet]. 2017 [citado 10 ago. 2021];5(1):71-78. doi: http://dx.doi.org/10.12662/2317-3076jhbs.v5i1.1054.p7178.2017. Acesso em: 3 nov. 2021.

${ }^{8}$ Kawasaki R, Nakao R, Ohnishi M. Contribution of social relationships to self-rated health among Japanese community-dwelling elderly. J. Rural Med. [internet]. 2018 [citado 10 ago. 2021];13(1):18-25. doi: https://doi.org/10.2185/jrm.2949. Acesso em: 3 nov. 2021.

${ }^{9}$ Harschel AK, Schaap LA, Iwarsson S, Horstmann V, Tomsone S. Self-rated health among very old people in European countries: An explorative study in Latvia and Sweden. Gerontol Geriatr Med. [Internet]. 2015 [citado 10 ago. 2021];1:1-10. doi: https://doi. org/10.1177/2333721415598432. Acesso em: 3 nov. 2021.

10 Lindemann IL, Reis NR, Mintem GC, Mendoza-Sassi RA. Autopercepção da saúde entre adultos e idosos usuários da Atenção Básica de Saúde. Ciênc. Saúde Colet. [Internet]. 2019 [citado 10 ago. 2021];24(1):45-52. doi: https://doi.org/10.1590/141381232018241.34932016. Acesso em: 3 nov. 2021.

${ }^{11}$ Medeiros SM, Silva LSR, Carneiro JA, Ramos GCF, Barbosa ATF, Caldeira AP. Fatores associados à autopercepção negativa da saúde entre idosos não institucionalizados de Montes Claros, Brasil. Ciênc. Saúde Colet. [Internet]. 2016 [citado 10 ago. 2021];21(11):33773386. doi: https://doi.org/10.1590/1413-812320152111.18752015. Acesso em: 3 nov. 2021.

12 Nascimento RCRM, Álvares J, Guerra Junior AA, Gomes IC, Silveira MR, Costa EA et al. Polypharmacy: a challenge for the primary health care of the Brazilian Unified Health System. Rev. Saúde Pública. [Internet]. 2017 [citado 10 ago. 2021]; 51(suppl 2): 1s-12s. doi: https://doi.org/10.11606/S1518-8787.2017051007136. Acesso em: 3 nov. 2021.

${ }^{13}$ Cadogan CA, Ryan C, Hughes CM. Appropriate Polypharmacy and Medicine Safety: When Many is not Too Many. Drug Saf. [Internet]. 2016 [citado 10 ago. 2021];39(2):10916. doi: https://doi.org/10.1007/s40264-015-0378-5. Acesso em: 3 nov. 2021.

${ }^{14}$ Almeida OP, Ameida AS. Confiabilidade da versão brasileira da Escala de Depressão em Geriatria (GDS) versão reduzida. Arq. Neuro-Psiquiatr. [Internet]. 1999 [citado 10 ago. 2021]; 57(2B):421-426. doi: https://doi.org/10.1590/S0004-282X1999000300013. Acesso em: 3 nov. 2021.

${ }^{15}$ Lino VTS, Pereira SEM, Camacho LAB, Ribeiro Filho ST, Buskman S. Adaptação transcultural da Escala de Independência em atividades da vida diária (Escala de Katz). Cad. Saúde Pública. [Internet]. 2008 [citado 10 ago. 2021];24(1):103-112. doi: https://doi. org/10.1590/S0102-311X2008000100010. Acesso em: 3 nov. 2021. 
${ }^{16}$ Santos RL, Virtuoso Junior JS. Confiabilidade da versão brasileira da escala de atividades instrumentais da vida diária. Rev. Bras. Promoç. Saúde. [Internet]. 2008 [citado 10 ago. 2021]; 21(4):290-296. doi: http://dx.doi.org/10.5020/18061230.2008.p290. Acesso em: 3 nov. 2021

${ }^{17}$ Roedl K, Wilson L, Fine J. A systematic review and comparison of functional assessments of community-dwelling elderly patients. J Am Assoc Nurse Pract. [Internet]. 2016 [citado 10 ago. 2021];28(3):160-169. doi: https://doi.org/10.1002/2327-6924.12273. Acesso em: 3 nov. 2021.

${ }^{18}$ Gomes GC. Tradução, adaptação transcultural e exame das propriedades de medida a Escala "Performance - Oriented Mobility Assessment" (Poma) para uma amostra de idosos institucionalizados. Campinas. [Dissertação] - Universidade Estadual de Campinas, Unicamp; 2003.

${ }^{19}$ Karuka AH, Silva JAMG, Navega MT. Análise da concordância entre instrumentos de avaliação do equilíbrio corporal em idosos. Rev. Bras. Fisioter. [Internet]. 2011 [citado 10 ago. 2021];15(6);460-466. Disponível em: https://pesquisa.bvsalud.org/portal/resource/pt/lil-611341. Acesso em: 3 nov. 2021.

${ }^{20}$ Ciconelli RM, Ferraz MB, Santos W, Meinão I, Quaresma MR. Tradução para a língua portuguesa e validação do questionário genérico de avaliação de qualidade de vida SF36 (Brasil SF-36). Rev. Bras. Reumatol. [Internet]. 1999 [citado 10 ago. 2021];39(3):143150. Disponível em: https://pesquisa.bvsalud.org/portal/resource/pt/lil-296502. Acesso em: 3 nov. 2021.

${ }^{21}$ Cardoso FP, Gonçalves AK. Associação entre domínio de capacidade funcional (SF-36), medo de cair e histórico de quedas em idosos ativos. Ciênc. Movimento. [Internet]. 2016 [citado 10 ago. 2021];18(36):1-8. doi: https://doi.org/10.15602/1983-9480/ cmbs.v18n36p1-8. Acesso em: 3 nov. 2021.

${ }^{22}$ Oliveira BLCA, Thomaz EBAF, Silva RA. The association between skin color/race and health indicators in elderly Brazilians: a study based on the Brazilian National Household Sample Survey (2008) Associação da cor/raça aos indicadores de saúde. Cad. Saúde Pública. [Internet]. 2014 [citado 10 ago. 2021];30(7):1438-1452. doi: https:// doi.org/10.1590/0102-311X00071413. Acesso em: 3 nov. 2021.

${ }^{23}$ Santos EC, Couto BM, Bastone AC. Fatores associados à autoavaliação negativa da saúde em idosos cadastrados nas Unidades Básicas de Saúde. ABCS Health sci. [Internet]. 2018 [citado 10 ago. 2021];43(1):47-54. doi: https://doi.org/10.7322/abcshs. v43i1.999. Acesso em: 3 nov. 2021.

${ }^{24}$ Muniz EA, Aguiar MFS, Brito MCC, Freitas CASL, Moreira ACA, Araújo CRC. Desempenho nas atividades básicas da vida diária de idosos em Atenção Domiciliar na Estratégia Saúde da Família. Rev Kairós Gerontol. [Internet]. 2016 [citado 10 ago. 2021];19(2);133146. doi: https://doi.org/10.23925/2176-901X.2016v19i2p133-146. Acesso em: 3 nov. 2021.

${ }^{25}$ Pereira LC, Figueiredo MLF, Beleza CMF, Andrade EMLR, Silva MJ, Pereira AFM, et al. Fatores preditores para incapacidade funcional de idosos atendidos na atenção básica. Rev. Bras. Enferm. [Internet]. 2017 [citado 10 ago. 2021];70(1):112-118. doi: https:// doi.org/10.1590/0034-7167-2016-0046. Acesso em: 3 nov. 2021.

${ }^{26}$ Ferreira AP. Capacidade e desempenho para a realização das atividades básicas de vida diária (básicas e instrumentais) em idosos dependentes. Rev. Baiana Saúde Pública. [Internet]. 2015 [citado 10 ago. 2021];39(1):25-37. doi: https://doi.org/10.22278/23182660.2015.v39.n1.a761. Acesso em: 3 nov. 2021.

27 Pampolim G, Lourenço C, Silva VG, Coelho MCR, Sogame LCM. Prevalence and factors associated with functional dependency in homebound elderly. J. Hum. Growth Dev. [internet]. 2017 [citado 10 ago. 2021];27(2):235-243. doi: https://dx.doi.org/10.7322/ jhgd.127747. Acesso em: 3 nov. 2021.

${ }^{28}$ Carneiro JA, Gomes CAD, Durães W, Jesus DR, Chaves KLL, Lima CA et al. Autopercepção negativa da saúde: Prevalência e fatores associados entre idosos assistidos em centro de referência. Ciênc. Saúde coletiva. [Internet]. 2020;25(3):909-918. doi: https://doi. org/10.1590/1413-81232020253.16402018. Acesso em: 3 nov. 2021. 
${ }^{29}$ Abreu DROM, Azevedo RCS, Silva AMC, Reiners AAO, Abreu HCA. Fatores associados à recorrência de quedas em uma coorte de idosos. Ciênc. Saúde Colet. [Internet]. 2016 [citado 10 ago. 2021];21(11):3439-3446. doi: https://doi.org/10.1590/1413812320152111.21512015. Acesso em: 3 nov. 2021.

${ }^{30}$ Vagetti GC, Moreira NB, Barbosa Filho VC, Oliveira V, Cancian CF, Mazzardo O et al. Domínios da qualidade de vida associados à percepção de saúde: um estudo com idosas de um programa de atividade física em bairros de baixa renda de Curitiba, Paraná, Brasil. Ciênc. saúde coletiva. [Internet]. 2013 [citado 10 ago. 2021];18(12):3483-3493. doi: https://doi.org/10.1590/S1413-81232013001200005. Acesso em: 3 nov. 2021.

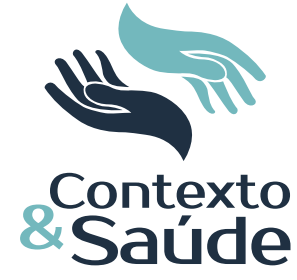

